\title{
Management of Sepsis: Recent Advancement
}

\author{
MI PATWARY ${ }^{a}$, MZJ BARI $^{b}$, IT ISHA $^{c}$
}

\begin{abstract}
Summary:
Sepsis is a systemic, deleterious host response to infection leading to acute organ dysfunction secondary to documented or suspected infection and septic shock i.e. sepsis plus hypotension not reversed with fluid resuscitation. Severe sepsis is a major healthcare problem with an extremely high mortality rate of $30-60 \%$ and it is one of the most common reasons for critically ill patients to be admitted to an intensive care unit (ICU). Excessive inflammation and coagulation and suppression of fibrinolysis are the hallmarks of Sepsis. Our medical concern is to manage sepsis and to prevent
\end{abstract}

\section{Introduction:}

Sepsis is defined as a severe infection with some degree of associated organ dysfunction (i.e. presence of SIRS in the setting of infection) ${ }^{1}$ and affects a large proportion of the critically ill population. In recent years, it has become clear that perhaps the most important as-pect of the management of patients with sepsis is early recogni-tion so that administration of antibiotics, source control measures and effective resuscitation strategies can be started as soon as possible after onset. Early management with adequate antimicro-bials and rapid resuscitation to restore and stabilize hemodynam-ic status has been shown to be associated with improved out-comes. ${ }^{1-5}$ However, diagnosis of sepsis is not always easy, especially in critically ill patients with other conditions and signs and symptoms that can mimic severe infection. $4-6$

The morbidity and mortality of sepsis in low- and middleincome countries (LMICs) are believed to be disproportionately high, due to environmental degradation, widespread malnutrition, and higher rates of bacterial, parasitic, fungal and HIV infection. ${ }^{7-10}$ In

a. Prof. Dr. Md. Ismail Patwary, Professor, Department of Medicine, Sylhet Women's Medical College.

b. Dr. Mohammad Zabed Jillul Bari, Assistant Professor, Department of Medicine, Sylhet MAG Osmani Medical College.

c. Dr. Ishrat Tahsin Isha, Assistant Registrar, Department of Medicine, Sylhet Women's Medical College.

Received: 9 Sept. 2015

Accepted: 4 Oct. 2016 multi organ failure (MOF). Making an early, accurate diagnosis of septic shock is the key to increasing survival rates. With no specific, effective anti-sepsis therapies available, management focuses on haemodynamic stabilization and rapid resuscitation, early source control, adequate and appropriate antibiotics, organ support and modulation of the septic responses are the cornerstones of treatment.

Key Words: Sepsis, severe sepsis, Septic shock.

(J Bangladesh Coll Phys Surg 2016; 34: 206-212)

an effort to reduce the risk of death from sepsis, the Surviving Sepsis Campaign (SSC) was initiated in 2002 from the collaboration of the European Society of Intensive Care Medicine (ESICM), the International Sepsis Forum (ISF), and the Society of Critical Care Medicine (SSCM). In 2004, the SSC produced the "Surviving Sepsis Campaign guidelines for management of severe sepsis and septic shock," one of the most recognized consensus statements regarding the treatment of sepsis (most recently updated in 2012). ${ }^{1}$

This review will discuss the epidemiology, pathophysiology, and diagnostic and therapeutic approach to patients with sepsis, severe sepsis, and septic shock in acute care settings.

\section{Epidemiology:}

Despite the documented impact of sepsis in developed countries, literature on its incidence, prevalence, and mortality in developing countries is sparse. ${ }^{8-11}$ The global burden of sepsis lies in LMICs. As a surrogate marker for sepsis, over $90 \%$ of worldwide deaths due to pneumonia, meningitis, and other infections occur in less developed nations (specific etiology usually gram positive or gram negative septicemia and rarely fungi, viruses). Globally, an estimated $70 \%$ of the 9 million annual neonatal and infant deaths are attributable to sepsis, and more than half of these occur in Asia and Sub- Saharan Africa. ${ }^{9,12-13}$ 


\section{Pathophysiology:}

In sepsis multiple organ dysfunction syndrome(MODS)[Ischemic encephalopathy, diminished myocardial contractility, ARDS, ATN, DIC, Hemorrhagic enteropathy, Cortical cell lipid depletion in adrenal gland etc.] appears to result from a cascade of organism-related factors, circulating immune or inflammatory mediators, diffuse endothelial cell injury, disturbed hemostasis or tissue/organ hypoperfusion, and microcirculatory abnormalities (microaggregation of microthrombi). In patients with severe sepsis, derangements of inflammation and coagulation are tightly linked. Free radicals, superoxide's, proteolytic enzymes are also directly involved the pathophysiology and occasionally impaired the cellular or the organ function. ${ }^{1-3}$

Table-I

\section{SIRS criteria. ${ }^{1}$}

\section{Presence of two or more of the following;}

1. Temperature $\geq 38^{0} \mathrm{C}\left(100.4^{0} \mathrm{~F}\right)$ or $\leq 36^{0} \mathrm{C}\left(96.8^{0} \mathrm{~F}\right)$

2. Heart rate $>90 / \mathrm{min}$

3. Respiratory rate $>20 / \mathrm{min}$ or $\mathrm{PaCO} 2<32 \mathrm{~mm} \mathrm{Hg}$

4. White blood cell count $\quad>12,000 / \mu \mathrm{L}-1$ or $<4000 / \mu \mathrm{L}-1$

Table-II

\section{Diagnostic Criteria for Sepsis: ${ }^{1}$}

\section{General variables}

Fever $\left(>38.3^{\circ} \mathrm{C}\right)$

Hypothermia (core temperature $<36^{\circ} \mathrm{C}$ )

Heart rate $>90 / \mathrm{min}$

Tachypnea

Altered mental status

Significant edema or positive fluid balance $(>20 \mathrm{~mL} / \mathrm{kg}$ over $24 \mathrm{hr}$ )

Hyperglycemia (plasma glucose $>140 \mathrm{mg} / \mathrm{dL}$ or $7.7 \mathrm{mmol} / \mathrm{L}$ ) in the absence of diabetes

\section{Inflammatory variables}

Leukocytosis (WBC count $>12,000 \mathrm{iL}-1$ )

Leukopenia (WBC count $<4000$ iL-1)

Normal WBC count with greater than $10 \%$ immature forms

Increased plasma C-reactive protein

Increased plasma procalcitonin

Hemodynamic variables

Arterial hypotension ( $\mathrm{SBP}<90 \mathrm{~mm} \mathrm{Hg}, \mathrm{MAP}<70 \mathrm{~mm} \mathrm{Hg}$, or an SBP decrease $>40 \mathrm{~mm} \mathrm{Hg}$ in adults )

Organ dysfunction variables

Arterial hypoxemia $(\mathrm{Pao} 2 / \mathrm{Fio} 2<300)$

Acute oliguria (urine output $<0.5 \mathrm{~mL} / \mathrm{kg} / \mathrm{hr}$ for at least $2 \mathrm{hrs}$ despite adequate fluid resuscitation)

Creatinine increase $>0.5 \mathrm{mg} / \mathrm{dL}$ or $44.2 \mathrm{imol} / \mathrm{L}$

Coagulation abnormalities (INR $>1.5$ or aPTT $>60 \mathrm{~s}$ )

Paralytic Ileus

Thrombocytopenia (platelet count $<100,000 \mathrm{iL}-1$ )

Hyperbilirubinemia (plasma total bilirubin $>4 \mathrm{mg} / \mathrm{dL}$ or $70 \mathrm{imol} / \mathrm{L}$ )

\section{Tissue perfusion variables}

Hyperlactatemia $(>1 \mathrm{mmol} / \mathrm{L})$

Decreased capillary refill 


\section{Table-III}

\section{Severe Sepsis ${ }^{1}$}

Sepsis-induced hypotension

Lactate above normal limit (more than $1 \mathrm{mmol} / \mathrm{L}$ )

Urine output $<0.5 \mathrm{~mL} / \mathrm{kg} / \mathrm{hr}$ for more than $2 \mathrm{hrs}$ despite adequate fluid resuscitation

Acute lung injury with Pao2/Fio $2<250$ in the absence of pneumonia as infection source

Acute lung injury with Pao2/Fio2 $<200$ in the presence of pneumonia as infection source

Creatinine $>2.0 \mathrm{mg} / \mathrm{dL}(176.8 \mathrm{imol} / \mathrm{L})$

Bilirubin $>2 \mathrm{mg} / \mathrm{dL}(34.2 \mathrm{imol} / \mathrm{L})$

Platelet count $<100,000 \mathrm{iL}$

Coagulopathy (INR $>1.5$ )

\section{Clinical assessment:}

During history taking, the focus should be on detecting risk factors for infection (such as age, chronic disease, immunosuppressive drugs, AIDS, preexisting comorbidities i.e. DM, Renal failure, bleeding disorder etc.).Caution should be advised in geriatric patients, as they may not be able to communicate traditional symptoms (e.g. dysuria in occult urinary tract infections). The physical examination should be used to identify possible foci of source control. A critical action at this point is the measurement, documentation, and evaluation of vital signs, including temperature, blood pressure (BP), heart rate (HR), respiratory rate (RR) and oxygen saturation (if below $90 \%$ then supplemental Oxygen should be immediately applied). Repeated recording of these parameters will be used to evaluate clinical improvement or deterioration and trigger specific interventions. ${ }^{1,14-15}$

Consistently analyzing the vital signs for the presence of SIRS criteria in any possible patient with sepsis will aid in the early recognition of critical illness. Importantly, vital sign derangements may be absent early in elderly patients. Specific physical exam findings that are predictive of sources of infection include indwelling devices (e.g., intravascular or urinary catheters), rales, abdominal tenderness, and evidence of CNS infection. A cardiovascular and volume status assessment, including auscultation, mucous membranes, skin colour and turgor, peripheral pulses, capillary refill and edema should be undertaken at this stage as well. ${ }^{1,14-15}$

\section{Investigations:}

Initial laboratory and radiographic testing is aimed at locating a source of infection and identifying evidence of organ dysfunction.

\section{Common laboratory studies include: ${ }^{1-3,14-17}$}

- Complete blood count (white blood cell count including a differential of subtypes and measure of bands, haemoglobin and haematocrit, platelets),

- Chemistries(electrolytes, bicarbonate, creatinine, glucose),

- Prothrombin time (PT)

- Liver transaminases, bilirubin,

- Arterial or venous blood gas analysis with the inclusion of a serum lactate level,

- Urinalyses are of high-yield, particularly in patients older than 65

- Chest radiography is to identify sources of pulmonary infection and causes of respiratory distress,

- Cultures as clinically appropriate before antimicrobial therapy with no significant delay ( $>45$ minutes) in the start of antimicrobials. At least 2 sets of blood cultures (both aerobic and anaerobic bottles) be obtained before antimicrobial therapy

- Urine, pus, ascetic fluid, pleural fluid, may be sent for culture where appropriate

\section{Treatment and management}

General management: The key principles of management of sepsis include early recognition, titrated fluid resuscitation, adequate source control, obtain blood cultures prior to administration of antibiotics, administer broad spectrum antibiotics, and organ support. Prompt recognition of the septic patient is critical, and early 
localization along the sepsis spectrum of illness helps to define the early goals of management. A key distinction should be made between sepsis and severe sepsis/septic shock (SS/SS), the latter of which are the focus of the Surviving Sepsis Campaign guidelines. It is recommended that patients with $\mathrm{SS} / \mathrm{SS}$ should undergo a protocol-driven approach which is suggested by Rivers study in 2001 and supported by meta analysis in 2008. ${ }^{18-19}$ It is a goal-directed approach to therapy of SS/SS patients (early goal-directed therapy, EGDT) and demonstrates a $16 \%$ absolute reduction in hospitalmortality ${ }^{18-19}$ However, the ProCESS trial, a recent multicenter randomized trial of over 1300 patients demonstrated no such benefit. ${ }^{20}$

Initial resuscitation: The goals during the first $6 \mathrm{hrs}$ of resuscitation: ${ }^{1}$

a) Central venous pressure $8-12 \mathrm{~mm} \mathrm{Hg}$

b) Mean arterial pressure (MAP) e" $65 \mathrm{~mm} \mathrm{Hg}$

c) Urine output e" $0.5 \mathrm{~mL} / \mathrm{kg} / \mathrm{hr}$

d) Central venous (superior vena cava) or mixed venous oxygen saturation $70 \%$ or $65 \%$, respectively

In patients with elevated lactate levels targeting resuscitation to normalize lactate. ${ }^{1}$

In such instances, a goal-directed approach to therapy is recommended by consensus guidelines with measurable endpoints at the 3 - and 6-h marks. ${ }^{1}$ Values significantly below this may be suggestive of hypovolaemia and the potential need for additional fluid resuscitation. This intervention alone has been shown to reduce mortality. ${ }^{21-22}$ In ProCESS trial; aggressive fluid resuscitation (an average of $4.4 \mathrm{~L}$ in the first $6 \mathrm{~h}$ in this study) causes reduce mortality. ${ }^{20}$

Fluid selection: Crystalloids as the initial fluid of choice in the resuscitation of severe sepsis and septic shock. Albumin is needed in the fluid resuscitation of severe sepsis and septic shock when patients require substantial amounts of crystalloids. Initial fluid challenge or goal in patients with sepsis-induced tissue hypoperfusion is to achieve a minimum of $30 \mathrm{~mL} / \mathrm{kg}$ of crystalloids. $^{1,21-22}$

Vasopressors: Vasopressor therapy initially to target a mean arterial pressure (MAP) of $65 \mathrm{~mm} \mathrm{Hg}$ .Norepinephrine as the first choice vasopressor. Epinephrine is added when an additional agent is needed to maintain adequate blood pressure. Vasopressin 0.03 units/minute can be added to norepinephrine (NE) with intent of either raising MAP in refractory hypotension and have beneficial hemodynamic and renal function effects $^{6}$.Dopamine is an alternative vasopressor to norepinephrine only in highly selected patients. Lowdose dopamine should not be used for renal protection ${ }^{1}$

Table-IV

Surviving Sepsis Campaign Bundles ${ }^{1}$

To be completed within 3 hours:

1) Measure lactate level

2) Obtain blood cultures prior to administration of antibiotics

3) Administer broad spectrum antibiotics

4) Administer $30 \mathrm{~mL} / \mathrm{kg}$ crystalloid for hypotension or lactate e" $4 \mathrm{mmol} /$ L to be completed within 6 hours:

5) Apply vasopressors (for hypotension that does not respond to initial fluid resuscitation) to maintain a mean arterial pressure (MAP) $>65 \mathrm{~mm} \mathrm{Hg}$

6) In the event of persistent arterial hypotension despite volume resuscitation (septic shock) or initial lactate $>4$ $\mathrm{mmol} / \mathrm{L}(36 \mathrm{mg} / \mathrm{dL})$ :

- Measure central venous pressure (CVP)

- Measure central venous oxygen saturation (ScvO2)

7) Remeasure lactate if initial lactate was elevated

*Targets for quantitative resuscitation included in the guidelines are CVP of $>8 \mathrm{~mm} \mathrm{Hg}, \mathrm{ScvO} 2$ of $>70 \%$, and normalization of lactate 


\section{Inotropic Therapy}

A trial of dobutamine infusion 20 micrograms $/ \mathrm{kg} / \mathrm{min}$ be administered or added to vasopressor in the presence of myocardial dysfunction or signs of hypoperfusion. ${ }^{1}$

In a recent, multicenter trial comparing norepinephrine and dopamine, both were equally efficacious at reversing hypotension and there was no significant difference in mortality, though there were more arrhythmias in the dopamine group. ${ }^{1}$

\section{Antimicrobial Therapy}

Administration of effective intravenous antimicrobials within the first hour of recognition of septic shock and severe sepsis is the norm. Initial empiric anti-infective therapy should be one or more drugs that have activity against all likely pathogens (bacterial and/or fungal or viral) and that penetrate inadequate concentrations into tissues. Antimicrobial regimen should be reassessed daily. Before starting antimicrobials blood/urine culture should be sent. Combination empirical therapy for neutropenic patients with severe sepsis may be needed. Empiric combination therapy should not be administered for more than 3-5 days (till culture report comes) .Duration of therapy typically 7-10 days; longer courses may be appropriate in patients who have a slow clinical response. Antiviral therapy may be initiated as early as possible in patients with severe sepsis or septic shock of viral origin. ${ }^{1}$

The choice of empirical antibacterial therapy varies significantly based on patient characteristics. Important considerations include the most likely source(s) of infection, recent antibiotics (last 3 months), recent health care exposure (e.g., hospitalization), underlying chronic disease, local pathogens and drug resistance. Patients with recent antibiotic exposure have an elevated incidence of high-risk infections such as methicillinresistant Staphylococcus aureus (MRSA) and Pseudomonas aeruginosa, whereas those with recent healthcare exposure have increased likelihood of being colonized with extended spectrum beta-lactamase (ESBL)-producing bacteria. ${ }^{1}$

\section{Source Control}

A specific anatomical diagnosis of infection requiring consideration for emergent of source control. If intravascular access devices or indwelling catheter are a possible source of severe sepsis or septic shock, they should be removed promptly. When infected peripancreatic necrosis is identified, definitive intervention is best delayed until adequate demarcation of viable and nonviable tissues has occurred. Selective oral decontamination and selective digestive decontamination should be introduced to reduce the incidence of ventilator-associated pneumonia. 1,23

\section{Corticosteroids}

Intravenous hydrocortisone is not encouraged to treat adult septic shock patients if adequate fluid resuscitation and vasopressor therapy are able to restore hemodynamic stability. Corticosteroids were not shown to be beneficial in SS/SS and in fact, potentially worsened outcomes. However, a later study by Annane et al demonstrated that patients with vasopressorunresponsive septic shock when treated with low-dose hydrocortisone and fludrocortisones causes shock reversal and reduces mortality. However a follow-up large multicenter trial (CORTICUS), showed that septic shock patients who responded to vasopressors, did not show a survival benefit with low-dose steroids. 1, 24-28

\section{Blood Product Administration}

Once tissue hypoperfusion has resolved, target hemoglobin is $7.0-9.0 \mathrm{~g} / \mathrm{dL}$ in adults. In patients with severe sepsis, administer platelets when counts are $<10,000 / \mathrm{mm} 3$ in the absence of apparent bleeding. Prophylactic platelet transfusion is advised when counts are $<20,000 / \mathrm{mm} 3$ if there is a significant risk of bleeding. Platelet counts (e"50,000/mm3) are advised for active bleeding, surgery, or invasive procedures . ${ }^{1}$

\section{Glucose Control}

Insulin should be started when 2 consecutive blood glucose levels are $>10 \mathrm{mmol} / \mathrm{L}(>180 \mathrm{mg} / \mathrm{dl})$ in ICU patient with severe sepsis. Blood glucose values should be monitored every $1-2 \mathrm{hrs}$ until glucose values and insulin infusion rates are stable and then every $4 \mathrm{hrs}$ thereafter. ${ }^{1}$ In resource-limited settings, check blood glucose levels in all patients. In the presence of mild hyperglycaemia, one should be careful about precipitating hypoglycaemia by insulin which may be more harmful. ${ }^{2}$

\section{Renal Replacement Therapy}

Renal replacement therapies and intermittent hemodialysis may be required in patients with Bicarbonate Therapy. Sodium bicarbonate is not 
recommended in patients with hypoperfusion-induced lactic acidemia when $\mathrm{pH}$ is $\geq 7 .{ }^{1}$.

\section{Deep Vein Thrombosis Prophylaxis}

Patients with severe sepsis should receive prophylaxis against venous thromboembolism (VTE) with daily subcutaneous LMWH. Combination of pharmacologic therapy and intermittent pneumatic compression devices may be needed. ${ }^{1}$

\section{Stress Ulcer Prophylaxis}

$\mathrm{H} 2$ blocker or proton pump inhibitor should be given to patients with severe sepsis/septic shock with bleeding risk. $^{1}$

Mechanical Ventilation of Sepsis-Induced Acute Respiratory Distress Syndrome (ARDS)

Target tidal volume of $6 \mathrm{~mL} / \mathrm{kg}$ body weight is recommended in patients with sepsis-induced ARDS. Positive end-expiratory pressure (PEEP) should be applied to avoid alveolar collapse at end expiration. Mechanically ventilated sepsis patients should be maintained with the head of the bed elevated to 30-45 degrees to limit aspiration and to prevent ventilatorassociated pneumonia. ${ }^{1,29}$

The inflammatory response to sepsis can cause lung injury and the development of ARDS. In a landmark multicenter randomized trial of lung-injured patients, those who underwent mechanical ventilation had a $9 \%$ absolute reduction in mortality. ${ }^{29-30}$

Immunoglobulins and Selenium

Intravenous immunoglobulin and selenium is not recommended in adult patients with severe sepsis or septic shock. ${ }^{1}$

Sedation, Analgesia, and Neuromuscular blockade in Sepsis

Continuous or intermittent sedation should be minimized in mechanically ventilated sepsis patients. Neuromuscular blocking agents (NMBAs) should be avoided in the septic patient without ARDS. ${ }^{1}$

\section{Nutrition}

Initiate oral or enteral feedings rather than either complete fasting or only intravenous glucose within the first 48 hours. Avoid full caloric feeding in the first week rather low dose feeding (up to 500 calories per day), gradually increasing as tolerated is recommended. ${ }^{1}$

\section{Supporting evidence}

Administration of human recombinant activated protein $\mathrm{C}$ is no longer recommended. The drug failed to show a survival benefit for patients with severe sepsis and septic shock . ${ }^{1}$

\section{Conclusion:}

Severe sepsis is a major healthcare problem with an extremely high mortality rate of $30-60 \%$. Consensus guidelines exist with specific recommendations for a bundled approach to the treatment of septic patients, and in particular, those with SS/SS. Our medical concern is to manage sepsis and to prevent MOF. Early sepsis recognition (e.g. serum lactate measurement), optimization of oxygen delivery (e.g. fluid resuscitation and vasopressors) and infection treatment (e.g. appropriate antibiotics and infection control, preceded by blood cultures) may result in a significant reduction in mortality and morbidity.

\section{References:}

1. Dellinger RP, Levy MM, Rhodes A, Annane D, Gerlach H, Opal SM, et al. Surviving sepsis campaign: international guidelines for management of severe sepsis and septic shock. Crit Care Med 2013; 41:580-637.

2. Angus DC, van der Poll T. Severe Sepsis and Septic Shock. N Engl J Med 2013; 369:840-51

3. Levy MM, Fink MP, Marshall JC, Abraham E, Angus D, Cook D,et al. $2001 \mathrm{SCCM} / \mathrm{ESICM} / \mathrm{ACCP} / \mathrm{ATS} / \mathrm{SIS}$ international sepsis definitions conference. Crit Care Med 2003; 31: 1250-56.

4. American College of Chest Physicians/Society of Critical Care Medicine Consensus Conference: definitions for sepsis and organ failure and guidelines for the use of innovative therapies in sepsis. Crit Care Med 1992; 20:864-74.

5. Angus DC, Linde-Zwirble WT, Lidicker J, Clermont G, Carcillo J, Pinsky MR et al. Epidemiology of severe sepsis in the United States: analysis of incidence, outcome, and associated costs of care. Crit Care Med 2001; 29:13031310 .

6. Nguyen HB, Smith D. Sepsis in the 21st century: recent definitions and therapeutic advances. Am J Emerg Med 2007; 25:564-571.

7. Cheng AC, West TE, Peacock SJ. Surviving sepsis in developing countries. Crit Care Med 2008; 36(8): 2487-8.

8. Cheng AC, West TE, Limmathurotsakul D, Peacock SJ. Strategies to reduce mortality from bacterial sepsis in adults in developing countries. PLoS Med 2008; 5: $175-80$ 
09. Baelani I,Jochberger S, Laimer T,OtienoD,Kabutu J, Wilson I, et.al. Availability of critical care resources to treat patients with severe sepsis or septic shock in Africa: a self-reported, continent-wide survey of anaesthesia providers. Crit Care 2011; 15:R10.

10. Black RE, Cousens S,Johnson HL, LawnJE, Rudan I, Bassani DG, et al. Global, regional, and national causes of child mortality in 2008: a systematic analysis. Lancet 2010; 375 (9730): 1969-87.

11. Zambon M, Ceola M, Almeida-de-Castro R, Gullo A, Vincent JL. Implementation of the surviving sepsis campaign guidelines for severe sepsis and septic shock: we could go faster. J Crit Care 2008; 23:455-60.

12. Jawad I, Lukšiæ I, Rafnsson SB. Assessing available information on the burden of sepsis: global estimates of incidence, prevalence and mortality. J Global Health 2012; 2: 010404.

13. Dünser MW, Festic E, Dondorp A, Kissoon N, Ganbat T, KwizeraA, et al. Recommendations for sepsis management in resource-limited settings. Intensive Care Med 2012; 38:557-74.

14. Cohen J, Brun-Buisson C, Torres A, Jorgensen J. Diagnosis of infection in sepsis: an evidence-based review. Crit Care Med 2004; 32(11 Suppl.):S466-94.

15. Maki DG, Kluger DM, Crnich CJ. The risk of blood stream infection in adults with different intravascular devices: a systematic review of 200 published prospective studies. Mayo Clin Proc 2006; 81:1159-71.

16. Lamy B, Roy P, Carret G, Flandrois JP, Delignette-Muller ML. What is the relevance of obtaining multiple blood samples for culture? A comprehensive model to optimize the strategy for diagnosing bacteremia. Clin Infect Dis 2002; 35:842-50.

17. Nguyen HB, Kuan WS, Batech M, Shrikhande P, Mahadevan M, Li CH, et al. Outcome effectiveness of the severe sepsis resuscitation bundle with addition of lactate clearance as a bundle item: a multi-national evaluation. Crit Care 2011; 15:R229.

18. Rivers E, Nguyen B, Havstad S, Ressler J, Muzzin A, KnoblichB, et al. Early goal-directed therapy in the treatment of severe sepsis and septic shock. N Engl J Med 2001; 345: 1368-77.

19. Jones AE, Brown MD, Trzeciak S, Shapiro NI, Garrett JS, Heffner AC, et al. The effect of a quantitative resuscitation strategy on mortality in patients with sepsis: a meta-analysis. Crit Care Med 2008; 36: 2734-9.
20. ProCESS Investigators. Yealy DM, Kellum JA, Huang DT, Barnato AE, Weissfeld LA, Pike F, et al. A randomized trial of protocol-based care for early septic shock. N Engl J Med 2014; 370: 1683-93.

21. Vincent JL, Gerlach H. Fluid resuscitation in severe sepsis and septic shock: an evidence-based review. Crit Care Med 2004; 32:S451-54.

22. Marik PE, Monnet X, Teboul JL. Hemodynamic parameters to guide fluid therapy. Ann Intensive Care $2011 ; 1: 1$.

23. Marshall JC, Maier RV, Jimenez M, Dellinger EP, et al. Source control in the management of severe sepsis and septic shock: an evidence-based review. Crit Care Med 2004; 32(11 Suppl.): S513-26.

24. Guidet B, Martinet O, Boulain T, Philippart F, Poussel JF, Maizel J, et al. Assessment of hemodynamic efficacy and safety of $6 \%$ hydroxyethyl starch $130 / 0.4$ vs. $0.9 \% \mathrm{NaCl}$ fluid replacement in patients with severe sepsis: the CRYSTMAS study. Crit Care 2012; 16: R94.

25. Perner A, Haase N, Guttormsen AB, Tenhunen J, Klemenzson G, Åneman A, et al. Hydroxyethyl starch 130/0.42 versus Ringer's acetate in severe sepsis. N Engl J Med 2012; 367: 124-34.

26. Cronin L, Cook DJ, Carlet J, Heyland DK, King D, Lansang MA, et al. Corticosteroid treatment for sepsis: a critical appraisal and meta-analysis of the literature. Crit Care Med 1995; 23:1430-9.

27. Annane D, Sébille V, Charpentier C, Bollaert PE, François B, Korach JM, et al. Effect of treatment with low doses of hydrocortisone and fludrocortisone on mortality in patients with septic shock. JAMA 2002; 288:862-71.

28. Sprung CL, Annane D, Keh D, Moreno R, Singer M, FreivogelK, et al. Hydrocortisone therapy for patients with septic shock. N Engl J Med. 2008 Jan 10; 358: 11124

29. Ventilation with lower tidal volumes as compared with traditional tidal volumes for acute lung injury and the acute respiratory distress syndrome. The Acute Respiratory Distress Syndrome Network. N Engl J Med. 2000 May 4; 342: 1301-1308.

30. Drakulovic MB, Torres A, Bauer TT, Nicolas JM, Nogué $\mathrm{S}$, Ferrer M, et al. Supine body position as a risk factor for nosocomial pneumonia in mechanically ventilated patients: a randomized trial. Lancet 1999 Nov 27; 354(9193):1851-58. 